\title{
A Counterexample to Hironaka's "Hard" Polyhedra Game
}

By

\author{
Mark SpIVAKovSKY*
}

In this paper, we give a counterexample to the "hard" polyhedra game, proposed by Hironaka some four years ago. Hironaka has proved that an affirmative solution of this game would imply the local uniformization theorem for an algebraic variety over an algebraically closed field of any characteristic. Although the answer turns out to be negative, we hope that this example may be useful for the problem of resolution of singularities: either for constructing a counterexample to the original problem or for modifying the rules of the game so that there will exist a winning strategy for the first player. The original, simpler version of the game, proposed by Hironaka in 1970 (see [2]) does have such a winning strategy (see [4] for a proof) which gives local uniformization in some special cases.

I am very grateful to Professor Hironaka for his patient advice and discussions, as well as his warm hospitality during my stay in Kyoto.

\section{Formulation of the Problem}

We are given a set $\Delta$ in the first quadrant $\mathbb{R}_{+}^{n}$ of $\mathbb{R}^{n}\left(\mathbb{R}_{+}^{n}=\left\{\left(x_{1}, \ldots, x_{n}\right) \in\right.\right.$ $\boldsymbol{R}^{n} \mid$ all $\left.\left.x_{i} \geq 0\right\}\right)$, which is the positive convex hull of a finite set of points, i.e., $\Delta$ is the convex hull of $\bigcup_{k=1}^{m}\left(V_{k}+\boldsymbol{R}_{+}^{n}\right)$, for some finite subget $\left\{V_{k}\right\}_{k=1}^{m} \subset \mathbb{R}_{+}^{n}$. (The same will also be denoted with a bracket: $\left.\Delta=\left[\left\{V_{k}\right\}_{k=1}^{m}\right]\right)$. Furthermore, all the coordinates of all of the $V_{k}$ are rational numbers whose denominators are bounded by some constant $N$ fixed throughout the game.

Communicated by H. Hironaka, October 9, 1981. Revised December 2, 1981.

* Research Institute for Mathematical Sciences, Kyoto University, 606 Kyoto Japan. Present address: Department of Mathematics, Harvard University, Cambridge, MA 02138, USA. 
Initially, we assume $\sum_{j=1}^{n} x_{j}>1$ for all $\left(x_{1}, \ldots, x_{n}\right) \in \Delta$.

Two people, $\mathrm{A}$ and $\mathrm{B}$, are playing the following game: A chooses a subset $\Gamma \subset\{1, \ldots, n\}$ such that $\sum_{j \in \Gamma} x_{j} \geq 1$ for all $\left(x_{1}, \ldots, x_{n}\right) \in \Delta$ (such $\Gamma$ is called permissible).

Then, B chooses some element $i \in \Gamma$ and modifies the set $\Delta$ to a set $\Delta^{*}$ by the following procedure: first, B selects a finite number of points $b=\left(b_{1}, \ldots, b_{n}\right)$ $\in \mathbb{R}_{+}^{n}$, all of whose coordinates are rational numbers with denominators bounded by $N$ as above, and for each of which there exists an $a=\left(a_{1}, \ldots, a_{n}\right) \in \Delta$ such that

(1) $b_{j}=a_{j} \quad$ for $j \notin \Gamma$ or $j<i$

(2) $b_{i}>a_{i}$

(3) $b_{j} \leqq a_{j}$ when $j \in \Gamma$ and $j>i$

(4) $\sum_{j=1}^{n} b_{j}=\sum_{j=1}^{n} a_{j}$.

$\Delta^{*}$ is then taken to be the positive convex hull of $\Delta U$ \{the selected points\}.

Note. The allowance of this modification $\Delta \mapsto \Delta^{*}$ is the only difference of the "hard" game from the original "simple" polyhedra game.

Then, let $\Delta^{\prime}=\sigma_{\Gamma, i}\left(\Delta^{*}\right)$, where $\sigma_{\Gamma, i}$ is the transformation of $\mathbb{R}^{n}$ sending $\left(x_{1}, \ldots, x_{n}\right)$ to $\left(x_{1}^{\prime}, \ldots, x_{n}^{\prime}\right)$ defined by

$$
\begin{aligned}
& x_{j}^{\prime}=x_{j} \quad \text { if } \quad j \neq i \\
& x_{i}^{\prime}=\sum_{j \in \Gamma} x_{j}-1 .
\end{aligned}
$$

$\Delta$ is replaced by $\Delta^{\prime}$ and the procedure is repeated. A wins, if after a finite number of moves there exists a point $\left(x_{1}, \ldots, x_{n}\right) \in \Delta$ such that $\sum_{j=1}^{n} x_{j} \leqq 1$.

The problem is: does there exist a winning strategy for $A$ ?

\section{III}

For $n \leqq 2$, the answer is affirmative, as proved by Hironaka [1], which gives the local uniformization for curves and surfaces. The counterexample below is for $n=3$.

$$
\text { Let } \Delta=\left[\left\{\left(\frac{1}{3}, \frac{1}{3}, \frac{2}{3}\right),\left(\frac{3}{2}, \frac{1}{3}, 0\right),\left(\frac{5}{6}, \frac{3}{2}, 0\right)\right\}\right]=\Delta_{1} \text {. }
$$

First move: A has no choice but $\Gamma=\{1,2,3\}$.

B chooses $i=2$ and modifies $\Delta_{1}$ to $\Delta_{1}^{*}=\left[\left\{\left(\frac{1}{3}, \frac{1}{3}, \frac{2}{3}\right),\left(\frac{3}{2}, \frac{1}{3}, 0\right)\right.\right.$, 
$\left.\left.\left(\frac{5}{6}, \frac{3}{2}, 0\right),\left(\frac{1}{3}, \frac{13}{6}, 0\right)\right\}\right]=\left[\left\{\left(\frac{1}{3}, \frac{1}{3}, \frac{2}{3}\right),\left(\frac{3}{2}, \frac{1}{3}, 0\right),\left(\frac{1}{3}, \frac{13}{6}, 0\right)\right\}\right]$.

That is, B "adds" the vertex $\left(\frac{1}{3}, \frac{13}{6}, 0\right)$ to $\Delta_{1}$, which satisfies conditions (1)-(4) above with $a=\left(a_{1}, a_{2}, a_{3}\right)=\left(\frac{1}{3}, \frac{3}{2}, \frac{2}{3}\right) \in \Delta_{1}$, according to the rules of the game.

Then, $\sigma_{\Gamma, i}\left(\Delta_{1}^{*}\right)=\Delta_{2}=\left[\left\{\left(\frac{1}{3}, \frac{1}{3}, \frac{2}{3}\right),\left(\frac{3}{2}, \frac{5}{6}, 0\right),\left(\frac{1}{3}, \frac{3}{2}, 0\right)\right\}\right]$. Note that this is the reflection of $\Delta_{1}$ with respect to the plane $x=y$.

Second move: A has no choice but $\Gamma=\{1,2,3\}$.

B chooses $i=1$, replaces $\Delta_{2}$ by $\Delta_{2}^{*}=\left[\left\{\left(\frac{1}{3}, \frac{1}{3}, \frac{2}{3}\right),\left(\frac{13}{6}, \frac{1}{3}, 0\right)\right.\right.$, $\left.\left.\left(\frac{1}{3}, \frac{3}{2}, 0\right)\right\}\right]$, which again is allowed by the rules. Then, $\sigma_{T, i}\left(\Delta_{2}^{*}\right)=\Delta_{1}$.

This is repeated forever. All through the game, $\mathrm{A}$ has only one permissible move, $\Gamma=\{1,2,3\}$, and $\mathrm{B}$ alternates between $i=1$ and $i=2$, with the set $\Delta$ alternating between the two configurations $\Delta_{1}$ and $\Delta_{2}$. Clearly, A cannot win.

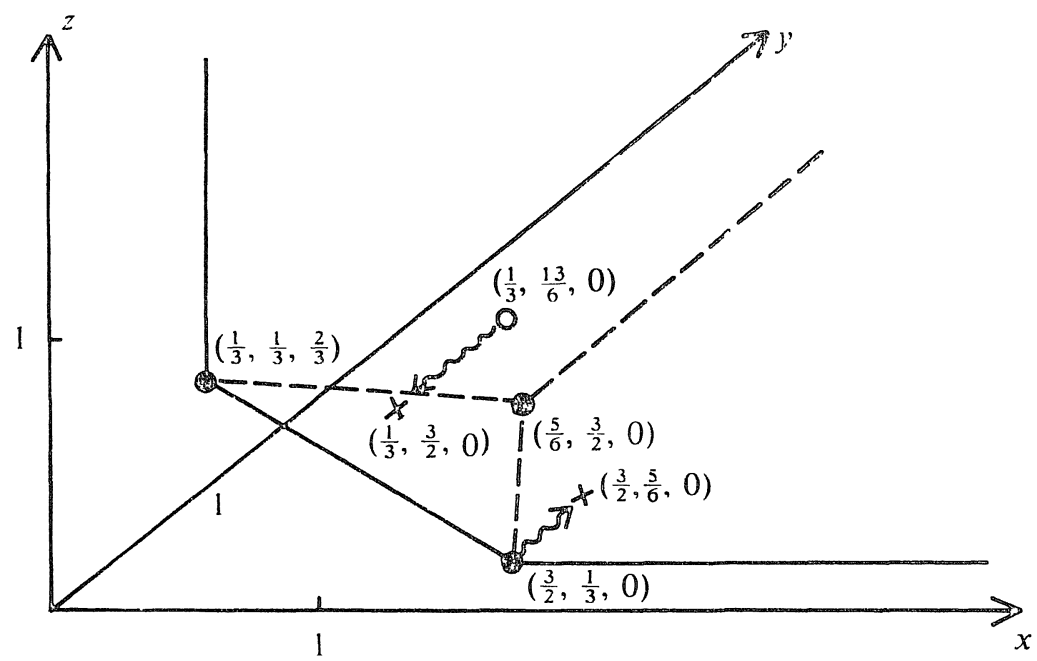

Here the black dots represent the vertices of $\Delta_{1}$, the crosses - the vertices of $\Delta_{2}$, the white circle - the vertex added by $B$ in the first move, and the wavy lines - the transformation $\sigma_{\Gamma, 2}$ of the first move.

\section{References}

[1] Hironaka, H., "Characteristic Polyhedra of singularities", J urnal of Mathematics of Kyoto University. Vol. 7, 1968, pp. 251-293. 
[2] Hironaka, H. "Schemes, etc.", Proc. 5th. Nordic Summer School in MATH, Oslo, 1970, pp. 291-313.

[ 3 ] Hironaka, H. "Certain numerical characters of singularities", Journal of Mathematics of Kyoto University, Vol. 10, 1970, pp. 151-187.

[4] Spivakovsky, M., "A solution to Hironaka's polyhedra game", to appear in the Šafarevich volume, Birkhäuser. 